\title{
Drill Bit Whirl Mitigation Analysis: An Under Actuated System Perspective
}

\author{
${ }^{1}$ F. Abdul Majeed, ${ }^{2}$ M. Karkoub, ${ }^{1}$ H. Karki, ${ }^{3}$ Y. L. Abdel Magid \\ ${ }^{1}$ Mechanical Engineering Department, The Petroleum institute, Abu Dhabi, U.A.E \\ ${ }^{2}$ Mechanical Engineering Department, Texas A\&M, Qatar \\ ${ }^{3}$ Electrical Engineering Department, The Petroleum institute, Abu Dhabi, U.A.E
}

\begin{abstract}
Lateral vibrations are the most destructive type of vibrations affecting rotary drilling. They are recognized by a phenomenon popularly known as drill bit whirl. The major causes leading to drill bit whirl are identified as the effect of a bend drill string or a manufacturing unbalance in the drill bit. This paper presents a detailed analysis of the drill bit whirl phenomenon by laboratory tests on a rotary drilling prototype built for the purpose. Practical methods which could control or limit these vibrations below the damaging threshold values are discussed in this paper. The paper also presents an explanation of the under actuation of the drill bit and hence its complex control objectives. A few typical under actuated system control solutions are presented, to analyze the scope of applying similar techniques to the drill bit whirl problem.
\end{abstract}

\section{Introduction}

Lateral vibrations are also known as bending vibrations. They cause the drill string to bend or break, resulting in severe damage to the drill bit and inhomogenous boreholes. Hence, lateral vibrations are the most destructive vibrations affecting the rotary drilling process [1]. The resultant phenomenon is termed bit whirl. Bit whirl is an eccentric motion of the drill bit in the borehole. It is usually accompanied by many other characteristics such as large frequent shocks and hitting of the entire bottom-hole-assembly (BHA) at the borehole [2].

\section{Factors leading to bit whirl}

Two major factors are identified leading to drill bit whirl. It could be due to either: (a) bend drill string or (b) an unbalanced drill bit. An in homogenous borehole could aggravate the conditions of whirling. These two different causes produced a similar phenomenon i.e.; the bit whirl. Jansen [3] stated that the bit whirling phenomenon occurs when drill bits have an imbalance in the drill bit design during manufacture or when there is a slight bend in the drill collars due to high lateral vibrations. Both of these imperfections cause the lateral vibrations to be predominant and cause bit whirl at higher operational ranges [4]. Warren et al. [5] analyzed commercially available bits and found that they have imbalances in the range of 2 to $10 \%$ with the $2 \%$ only for a very high commercially graded bit. An imbalance on the bit will cause it to stray away from its center of rotation during drilling. This will lead to high centrifugal force to be developed which will increase the side loading of the bit and eventually, result in hole enlargement and bit whirling.

\section{Analysis of Bit whirl experiments in literature}

Laboratory tests are conducted to analyze firsthand the drill bit whirl phenomenon. The laboratory arrangement was decided on the basis of the following analysis. Jansen [3] suggested that the dynamics of a bend drill string in forward whirl could be demonstrated by an unbalanced mass on the lower bit. Following this idea, unbalanced rotors were used to represent an eccentric drill pipe in the research by Dykstra [6] and C-Min Liao et al. [7].

Dykstra [6] conducted tests on drill collars and studied the effects of lateral shocks and backward whirl. The discussion was based on the fact that the source of vibration is the bit and hence the centrifugal forces developed when an unbalanced drill string is rotated can be one of the major sources of vibrations. C-Min Liao et al. [7] developed a reduced order model for a drill string system with a mass imbalance on the rotor. The tests were focused to analyze the trajectory of the bit under conditions of varying drill bit mass unbalance and operational velocities.

\section{Laboratory set-up}

The drill string in the laboratory arrangement is vertical, about $1 \mathrm{~m}$ long, and is made of carbon steel to ensure the material properties are as close as possible to the actual rig. In order to analyze the above discussions on lateral vibrations, various tests with different WOBs and operational velocity of the rotary table are conducted. Further to the analysis by Warren [5] on drill bit unbalance, test results (addition of $2.6 \%$ and $5.2 \%$ unbalance) are presented for the unbalanced drill bit experiments. 


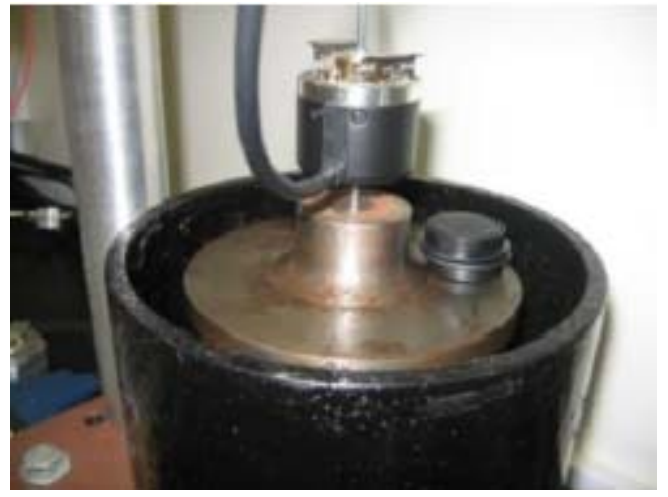

Figure 1. Laboratory set-up with unbalanced drill bit.

\subsection{Case 1: Ideal- Zero unbalance Condition}

Initially the drill string system is allowed to rotate freely (zero unbalanced mass). In this state the system can be compared to the state when the drill string rotates with an ideal drill bit (zero unbalance). It was noticed that at operational velocity of around 8 RPM, there existed some vibrations (Figure 2). These vibrations are the self exited vibrations, which arise in rotary drilling at very low speeds. However it was noted that when the speed was increased to 36 RPM, the self excited vibrations disappeared and vibrations similar to limit cycling appeared. At the operational speed of 51 RPM, the drill string rotated smoothly with less limit cycling and self exited vibrations.

The average operational speed of commercial drilling is in the range of 50-60 RPM. Analyzing the graphs for case 1 angular velocity data, it was noted that the upper rotary velocity lagged behind the command speed at lower speeds of 8 RPM and caught up with the command speed at 51 RPM. The lagging of the upper rotary at low speeds could be attributed to power dissipation in the elements. It is seen that the drill string upper and lower velocities followed the command speed more closely at higher speeds due to the fact that the power received by the system is much higher than the power dissipated in the system.

\subsection{Case 2: $2.6 \%$ Mass unbalanced drill bit condition}

The experiments prove that the upper rotary and drill bit velocities followed the command speed better than in the ideal condition (Figure 2). It was also noted that the self exited vibrations appeared similar to case 1 when rotated at low speeds, but they were less prominent due to the higher mass of the bit. When the operational speed was increased to 50 RPM, the system rotated at a speed slightly higher than the command speed, this could be attributed to the increase in nonlinear properties of the drill string.

\subsection{Case 3: $5.2 \%$ mass unbalanced drill bit}

Under higher unbalanced mass conditions, the system followed the command speed more closely at 39 RPM. Limit cycling vibrations were more prominent, and bit whirling was noticed at higher speeds (Figure 2). Figure 2a-c displays the command speeds applied to the system, the angular velocity data of the upper rotary and lower bit for the three cases discussed above.

a.

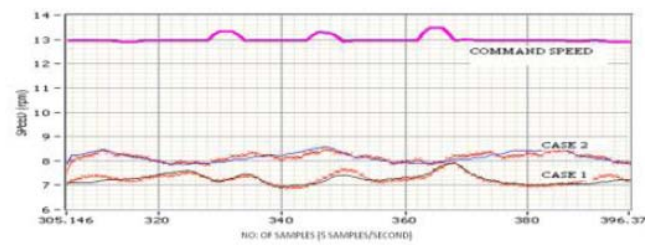

b.

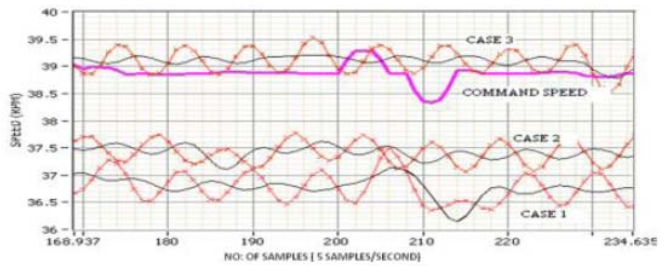

c.

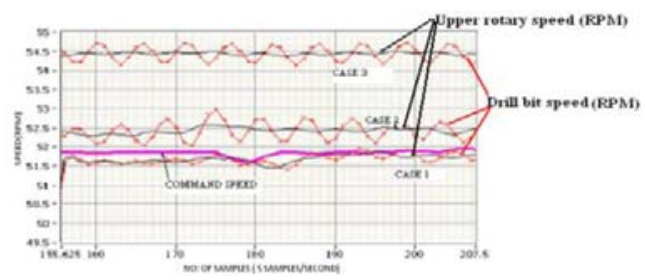

Figure 2. The plots of the experimental data under varying unbalanced mass conditions and operational velocities

\section{Rotary drilling rigs- an under actuated system?}

Oil drilling is a very important industry and the premature failure of drill strings is the most important problem the industry is facing. An under actuated system is characterized by the lesser number of actuated inputs when compared to the degrees of freedom. In order to better analyze the actuated elements and degrees of freedom in a rotary system, a closer analysis of the drilling process is required. Figure 3 displays a simple schematic of the rotary drilling rig. 


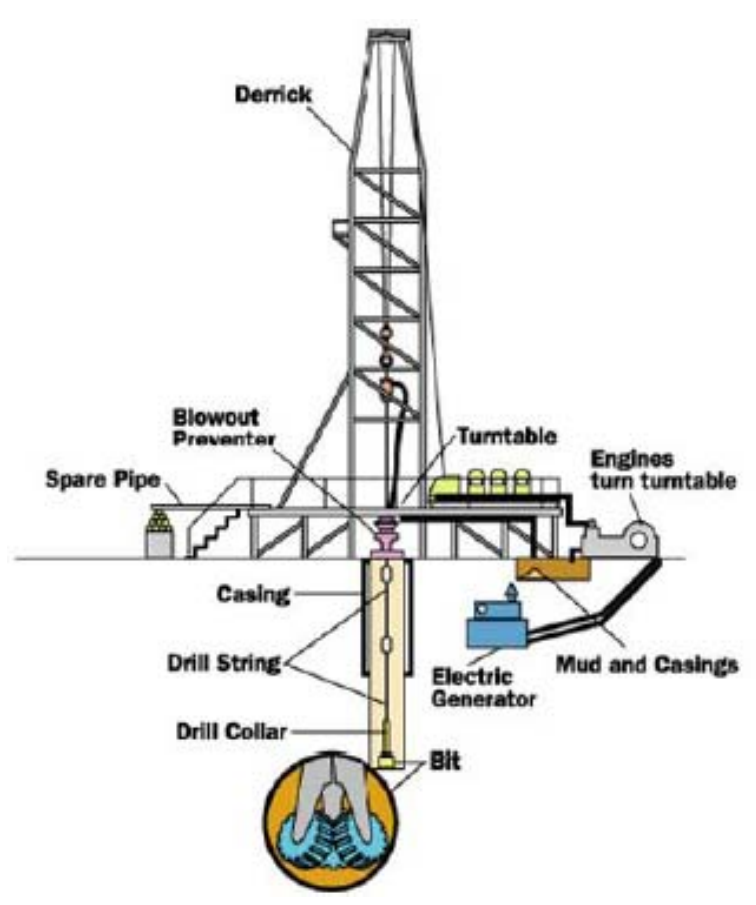

Figure 3. Schematic of a rotary oil rig [How stuff works, (2011)]

On closer analysis of the rotary drilling process, reveals that the upper rotor/ turntable is the only actuated part of the rotary drill rig. The drill pipes are connected together by joints which are not completely rigid. The drill bit is the part which is in contact with the borehole. The joints at the drill pipes lead to the driving force at the turntable in not being fully transmitted to the drill bit. Hence, affecting a change in the upper rotary will not affect a similar change at the drill bit. This situation can be partly overcome by using the borehole casing to hold the drill pipes in the borehole and decreasing the degree of freedom at the tool joints. The drill bit is not directly energized and hence there is lesser number of actuated elements when compared to the degrees of freedom for the system. Thus the drill string system can be considered as an under actuated system. Realizing the drill string as a special under actuated system, it now follows to investigate the possibility of applying control principles of under actuated system control to mitigate drill bit whirl. The above studies revealed the causes leading to drill bit whirl as physical in nature. The analysis of bit whirl dynamics proved that the controls solution should achieve two objectives: forcing the drill bit near to center of rotation and close following of the upper rotary velocity. Hence two typical under actuated systems which have similar control objectives are selected for further analysis.

\section{Two typical under-actuated system examples}

This section presents two typical under actuated system research. They have applied control techniques to achieve trajectory following and equilibrium positioning of the under actuated part.

\subsection{Case Study 1}

Block et al. [10] discusses the Pendubot, popular in under actuated systems. It is a two link, under actuated robotic mechanism (Figure 4). Link 1 is directly mounted on a motor shaft, and link 2 is coupled to link 1 by needle joint bearings. Both the links have full 360 degree freedom of rotational motion. The research tries to develop a controller to swing the mechanism from its open loop stable configuration to the unstable equilibrium points and then to catch the unactuated link (link2) and balance it there. This control is divided into two parts; swing up control and balancing control. The swing up control uses the method of partial feedback linearization. The balancing control uses linearizing the system and designing a full state feedback controller for that linearized model.

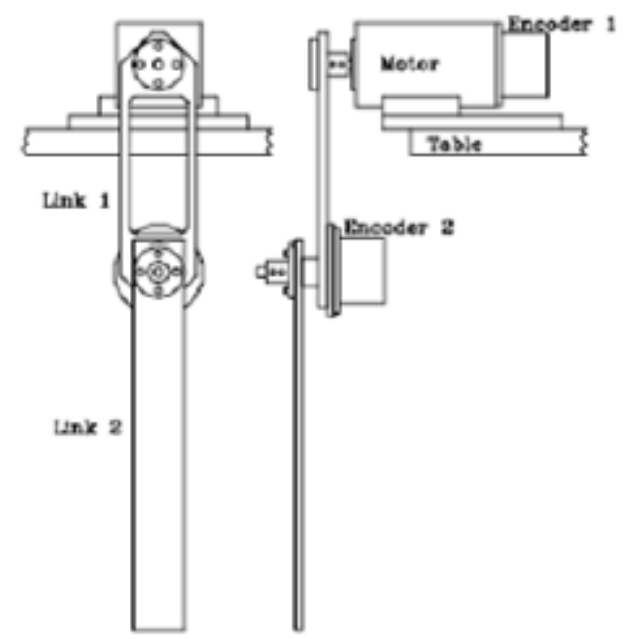

Figure 4. Schematic of Front and side perspective drawings of the Pendubot [10]

The equations of motion for the Pendubot can be found using Lagrangian dynamics. In matrix form the equations are:

$$
D(q) \ddot{q}+C(q, \dot{q}) \dot{q}+g(q)=\tau
$$

Where is the vector of torque applied to the links and $\dot{q}$ is the vector of joint angle positions. 
Partial Feedback Linearization needs position feedback from both link one and link two but takes into account the nonlinear effects of the linkage. Due to the under actuation of link two, only one DOF was linearized. Jerome had chosen to linearize about the collocated degree of freedom (link1). An outer loop control was designed to track a given trajectory for the linearized degree of freedom to achieve swing up control (figure 5a). For balancing control, the pendubot is balanced at equilibrium points at upright and mid balancing positions (Figure 5b). The Taylor series approximation was used to linearize the plant. The partial derivative matrices are evaluated at the equilibrium points to obtain linear models. LQR and pole placement techniques were used to design full state feedback controllers, $\mathrm{u}=-\mathrm{Kx}$ to achieve balancing control.

a.

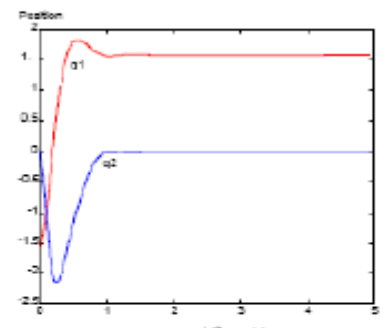

b.

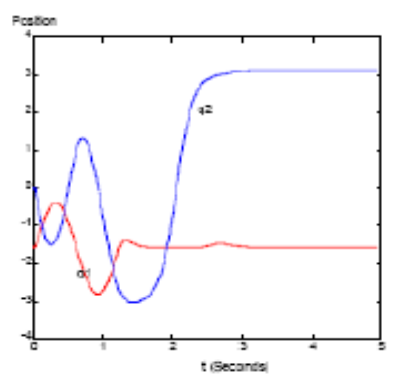

Figure 5(a) and 5(b): Swing up to the top position and swing up to the mid position [10].

\subsection{Case study 2:}

Blajer et al. [11] conducted under actuated system research on a system consisting of two rotating discs connected horizontally by a torsional string with the disc 2 as the under actuated element (Figure 6). Here the disc 2 is taken from rest to a 360 degrees movement and then rest/stops the movement.

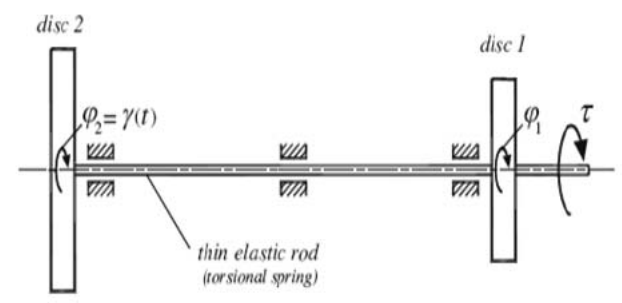

Figure 6. Schematic of Two degree of freedom system [11].
They defined a function $\mathrm{s}(\mathrm{t})$ to be an appropriately smooth reference function that models a rest-to-rest maneuver. Generally, for an n-degreeof-freedom under actuated system, described by a set of generalized coordinates $\mathrm{q}=[\mathrm{q} 1 \ldots \mathrm{qn}]^{\mathrm{T}}$ and actuated by $\mathrm{m}$ control inputs $\mathrm{u}=[\mathrm{u} 1 \ldots \mathrm{um}]^{\mathrm{T}}$, where $\mathrm{m}<\mathrm{n}$.

A motion or servo-constraint can be defined as,

$$
c(q, t) \equiv \phi(q)-v_{d}(t)
$$

which force the under actuated system to complete a partly specified motion or performance goals, $v_{d}(t)$. In the case studied, the specified motion $\phi_{2}=v(t)$ of disc 2 is actuated by the torque $\tau$ applied to disc 1 , and as such $n=2$ and $m=1$. The dynamic equations of the system are:

$$
\begin{aligned}
& J_{1} \ddot{\varphi}_{1}=c_{s}\left(\dot{\varphi}_{2}-\dot{\varphi}_{1}\right)+k_{s}\left(\varphi_{2}-\varphi_{1}\right)-\tau_{1 r e s}\left(\dot{\varphi}_{1}\right)+\tau \\
& J_{2} \ddot{\varphi}_{2}=-c_{s}\left(\dot{\varphi}_{2}-\dot{\varphi}_{1}\right)-k_{s}\left(\varphi_{2}-\varphi_{1}\right)-\tau_{2 r e s}\left(\dot{\varphi}_{2}\right)
\end{aligned}
$$

where $\mathrm{J} 1$ and $\mathrm{J} 2$ are the disc mass moments of inertia, ks and cs are the coefficients of rod stiffness and damping, and $\tau 1$ res and $\tau 2$ res are the resistance torques caused by friction and damping effects in the bearings. It is assumed that for the system the inputs affect the system dynamics linearly. A flatness based solution is proposed here on the condition that friction and damping effects are neglected, i.e. $c_{s}=0$ and $\tau_{\text {lres }}=\tau_{2 \text { res }}=0$ and the servo constraint is modeled to allow a specified motion in which a rest to rest maneuver is performed.

\section{Discussing applicability of under actuated control solutions to mitigate drill bit whirl}

Under actuated control objective is to control the position or a specified movement. But not velocity and position control together which is the control objective. In the swing up control of [10], the control objective is to make the linearized degree of freedom, link1, to track a suitable trajectory. Here, the controller objective is to control the pendubot only at the equilibrium positions. No external disturbance is affected at link 2. The positions of the links are not synchronized during the control operations.

Moreover the full state feedback controller is designed by initially linearizing the model in the required or set equilibrium positions. Thus control principle can only be applied to simple linearized models of the drill system, and cannot be proven practically on a nonlinear laboratory set-up. Moreover, the problem is to set two trajectories to be followed in the presence of unexpected disturbance. Also, the control is achieved in case study 1 for only a short period of time. Control can be well said to be near impossible to be achieved in the drilling system, which 
is operated in a highly unpredictable environment for an extended period of time.

The flatness based control solution of case study 2 is used to model a rest to rest maneuver. It should be noted that external friction/ damping force is not affecting the under actuated disc. Here again, the positions and velocities of the actuated and under actuated parts are not synchronized and are widely different at the time of application of the control law. Such a situation in the rotary drilling will the induce torsional vibration because of the friction developed at the drill bit by the bore hole and could result in more severe phenomena like backward whirl.

\section{Conclusion and Scope of Future Work}

From the above analysis, it can be concluded that the physical causes inducing drill bit whirl can be overcome only by using other physical solutions; like new drill bit designs or dampers near the BHA [5, 8, and9]. Research is underway to analyze control techniques to minimize the effect of the vibration aggravating sources like drill bit- bore hole friction, sudden / hard obstacles, etc.

\section{References}

[1] J. Zare, S.J. Hashemi and G. Rashed, "Finite Element Analysis of Drillstring Lateral Vibration", J. of Engineering and Applied Sciences, 2011 , Vol. 6, Issue: 1, pp. 64-70

[2] H. Spanos, J.C.R. Plácido, C. Wolter, "Consequences And Relevance Of Drillstring Vibration On Wellbore Stability", SPE/IADC Drilling Conference Held In Amsterdam, Holland, 9-11 March 1999.

[3] J.D. Jansen, "Non-Linear Rotor Dynamics As Applied To Oilwell Drillstring Vibrations", Journal Of sound And vibration ,1991, 147, pp.115-135.

[4] J. F. Brett, T.M. Warren, S. M. Behr, "Bit Whirl - A New Theory Of Pdc Bit Failure", SPE Drilling Engineering, Vol.5, N4, Dec 1990, pp.275-280.

[5] Warren, T M; Brett, J F; Sinor, L A ; "Development Of A Whirl-Resistant Bit", SPE Drilling Engng,1990, Vol.5, N4, pp.267-274.

[6] Dykstra, M.W., 1996. Nonlinear drill string dynamics. PhD Thesis, The University of Tulsa.

[7] C-Min Liao, B. Balachandran, M. Karkoub, "Drill String Dynamics: Reduced Order Models", ASME International Mechanical Engineering Congress and Exposition, Nov. 1319, 2009, Lake Buena Vista, Florida, USA.

[8] S.L. Chen, K. Blackwood; E. Lamine; "Field Investigation Of The Effects Of Stick-Slip, Lateral, And Whirl Vibrations On Roller-Cone Bit Performance", SPE Drilling \& Completion, Volume 17, Number 1 March 2002, pp. $15-20$.

[9] S.Johnson, "A New Method Of Producing Laterally Stable PDC Drill Bits", SPE Drilling \& Completion, Volume 23, Number 3, September 2008, pp. 314-324.

[10] D.J. Block, Mechanical design and control of the pendubot, MSc Thesis, University of Illinois, 1991.

[11] W. Blajer, K. Dziewiecki, K. Kołodziejczyk, Z. Mazur, "Inverse dynamics of under actuated mechanical systems: A simple case study and experimental verification", 2010,
Commun Nonlinear Sci Numer Simulation, Elsevier, Vol 16, Issue 5, pp.2265-2272. 